Original research article

\title{
Oral melatonin administration improves the objective and subjective sleep quality, increases 6-sulfatoxymelatonin levels and total antioxidant capacity in patients with fibromyalgia
}

\author{
M. Yolanda Castaño ${ }^{a}$, Maria Garrido ${ }^{\mathrm{a}}$, Jonathan Delgado-Adámez ${ }^{\mathrm{b}}$, Sara Martillanes ${ }^{\mathrm{b}}$, \\ M. Ángeles Gómez ${ }^{\mathrm{a}}$, Ana Beatriz Rodríguez ${ }^{\mathrm{a}, *}$ \\ ${ }^{a}$ University of Extremadura, Faculty of Science, Department of Physiology (Neuroimmunophysiology and Chrononutrition Research Group), Badajoz, Spain \\ ${ }^{\mathrm{b}}$ Technological Institute of Food and Agriculture (INTAEX), Gobierno de Extremadura, Av. Adolfo Suárez s/n, 06071 Badajoz, Spain
}

\section{A R T I C L E I N F O}

\section{Article history:}

Received 8 December 2016

Received in revised form 28 November 2017

Accepted 17 January 2018

Available online 1 February 2018

\section{Keywords:}

Fibromyalgia

Melatonin

Sleep quality

6-Sulfatoxymelatonin

Antioxidant capacity

\begin{abstract}
A B S T R A C T
Background/objective: Chronic pain, sleep disturbances and oxidative stress are implicated in the pathogenesis of fibromyalgia. The aim of this study was to assess the effect of melatonin supplementations on sleep quality, 6-sulfatoxymelatonin (aMT6-s) levels, as well as urinary and serum total antioxidant capacity (TAC) in patients with fibromyalgia.

Methods: Thirty three patients carried out the full study. A baseline period (10 days) was included in the experimental design to collect information about patients' initial status. Then, patients took different doses of melatonin, during 10 consecutive days each. Placebo was given during 10 days either before or between melatonin doses. Objective sleep quality was recorded by actigraphy whereas subjective sleep quality was measured by The Pittsburgh Sleep Quality Index. Quantification of aMT6-s and TAC was achieved by ELISA and colorimetric assay kits, respectively.

Results: Six out of seven sleep parameters evaluated by actigraphy were improved after the intake of melatonin as well as the subjective sleep quality. All the biochemical parameters measured were also elevated after the melatonin administration.

Conclusion: The daily intake of melatonin improved the sleep quality, increased the aMT6-s levels and the total antioxidant capacity in patients with fibromyalgia.

(c) 2018 Faculty of Health and Social Sciences, University of South Bohemia in Ceske Budejovice. Published by Elsevier Sp. z o.o. All rights reserved.
\end{abstract}

\section{Introduction}

The etiology of fibromyalgia (FM) is still unclear (Bellato et al., 2012). It is characterized by chronic widespread musculoskeletal pain. Besides sleep disturbances or poor-quality sleep (HitaContreras et al., 2014), anxiety, depression, and lack of concentration and memory (Giacomelli et al., 2013) are also common symptoms in patients with FM. Moreover, different studies have shown disruptions of circadian rhythms of certain biochemical parameters such as: melatonin, cortisol, serotonin or cytokine production (Mahdi et al., 2011). However, it is unknown if these abnormalities are cause or consequence of the disease.

Recently, it has been suggested that oxidative stress is implicated in the pathogenesis of FM (Fatima et al., 2015). Under oxidative stress conditions, excessive reactive oxygen species

\footnotetext{
* Author for correspondence: University of Extremadura, Faculty of Science, Department of Physiology, Avda. Elvas s/n, 06006 Badajoz, Spain.

E-mail address: moratino@unex.es (A.B. Rodríguez).
}

(ROS) production can damage biomolecules and cellular components (Akbas et al., 2014; Sánchez et al., 2015). In this context, increased ROS levels observed in blood mononuclear cells in patients with FM have been decreased by antioxidant actions (Akkuş et al., 2009; Nazıroğlu et al., 2010; Sartori et al., 2016), which reflect changes in markers of oxidative stress, including the Trolox equivalent antioxidant capacity (TEAC), whose level in serum has been widely used in the assessment of lipid peroxidation (Cordero et al., 2011).

Although pain is the most common symptom associated with FM, and the greatest therapeutic and research efforts have been focused on it, major sleep disturbances are also very common, representing up to $90 \%$ of patients. Moreover, a direct negative correlation between sleep and pain has been suggested (Chinn et al., 2016). In this line, it has also been proposed an important complex relationship between pain and sleep disturbance, since pain could disrupt sleep and, at the same time, sleep deprivation could enhance pain sensitivity (Diaz-Piedra et al., 2015; Spaeth et al., 2011). 
It is known that melatonin is involved in several physiological processes, including the regulation of circadian rhythms, pain, mood, and immune responses (De Zanette et al., 2014), and it is a well-known free radical scavenger and antioxidant (Tan et al., 1993). This indoleamine has been used in several clinical studies in FM patients with successful results (De Zanette et al., 2014; Wilhelmsen et al., 2011). Hence, Citera et al. (2000) have found that pain intensity was reduced and physical parameters were improved 30 days after the administration of $3 \mathrm{mg} /$ day of melatonin. In the same context, other studies have shown that doses of $5 \mathrm{mg} /$ day melatonin and $10 \mathrm{mg} /$ day, administered together with the usual medication of the patient, improved fatigue, mood, sleep quality and pain (De Zanette et al., 2014; Hussain et al., 2011).

Therefore, the purpose of this study was to assess the effect of the intake of different doses of melatonin (3, 6, 9, 12, $15 \mathrm{mg} /$ day) on sleep quality (objective and subjective assessments), the 6-sulfatoxymelatonin (aMT6-s) levels, the main urinary metabolite of melatonin, and the urinary and serum total antioxidant capacity in patients with severe fibromyalgia.

\section{Materials and methods}

\section{Participants}

Ninety seven patients with FM were initially recruited. Among them, 36 were finally enrolled in the study, according to the inclusion criteria: FM women diagnosed based on the American College of Rheumatology guidelines (Wolfe et al., 2010) aged more than 40 years-old, with $>70$ total score in Fibromyalgia Impact Questionnaire (FIQ), and not involved in physical/psychological therapies. There were three dropouts throughout the study due to family-related matters and disinterest in the adherence to the protocol of the study. For this reason, 33 out of 36 patients completed the full study. All participants kept their lifestyle habits related to medication, diet, sleep, and exercise throughout the study.

The study was approved by both, the Ethical Committee of the University of Extremadura (Badajoz, Spain) and the Ethical Committee of Servicio Extremeño de Salud, in accordance with the Declaration of Helsinki, the Council of Europe and the Universal Declaration of UNESCO on human rights, biomedicine and human genome. Informed consent was obtained from all participants.

\section{Experimental design}

The study had a longitudinal placebo-controlled design with 5 melatonin administration periods of 10 days each, separated by washout periods (10 days each). Before starting with the assay 10 days were left as basal period to collect information about patients' initial status. All the patients took different doses of melatonin (3, 6, 9, 12, $15 \mathrm{mg} /$ day). Each dose was taken as a single pill (Guinama, Puebla de Vallbona, Spain) at night, 30 min before going to bed during 10 consecutive days. Placebo (a rice starchcontaining pill) was administered at night (1 pill/day) during
10 days, either before (placebo period) or between (washout periods) the different doses of melatonin (Table 1 ).

Objective sleep assessment tool: actigraphy

Actigraphic monitoring was used to record and display the temporal patterns of the individuals' activity and rest (Actiwatch, Cambridge Neurotechnology Ltd., Cambridge, UK). Participants wore a wrist activimeter, on the non-dominant wrist that recorded activity throughout the whole assay. When the data collection period finished, the results were analyzed, with the sleep analysis (Cambridge Neurotechnology Ltd.) software package. The parameters analyzed with the software were: sleep efficiency (percentage of sleep time while the subject remains in bed); actual sleep time (assumed sleep minus awake time; determined by algorithms); wake bouts (the number of episodes of high activity during the period of sleep); total nocturnal activity (total pulses of activity during sleep); sleep latency (time from the individual lies down on bed to sleep onset); assumed sleep (the difference between the end and beginning of the period of sleep); immobility (minutes in which the subject has zero mobility).

\section{Subjective sleep assessment tool: Pittsburgh test}

The Pittsburgh Sleep Quality Index (PSQI) is a self-report questionnaire developed for measuring the subjective perception of sleep quality and patterns. This questionnaire includes 19 selfrated items divided in 7 components: sleep quality (1 item), sleep latency (2 items), sleep duration (1 item), habitual sleep efficiency (3 items), sleep disturbance (9 items), use of sleeping medication ( 1 item), and daytime dysfunction (2 items). The global score has a range from 0 to 21 . Higher score represents poorer subjective sleep quality (Hita-Contreras et al., 2014). This questionnaire was filled at the end of the different periods: baseline, placebo, melatonin administrations and washout periods.

\section{Urinary 6-sulfatoxymelatonin levels (aMT6-s)}

First-void morning urines were collected at the end of basal and placebo periods, and both and at the end of the administration of each different dose of melatonin and washout periods. The samples were stored at $-80{ }^{\circ} \mathrm{C}$ until biochemical assay. The urinary metabolite aMT6-s was quantified using a commercial enzyme-linked immunoabsorbent assay kit (DRG ${ }^{\circledR}$ MelatoninSulfate (EIA-1432), USA) according to the manufacturer's instructions.

To adjust for variation in the dilution of urine, 6-sulfatoxymelatonin was expressed as urinary 6-sulfatoxymelatonin/urine creatinine ratio. Creatinine concentration was determined by means of the Jaffe test.

\section{The total antioxidant capacity in urine samples}

First-void morning urines were collected both, at the end of basal and placebo periods, and at the end of the administration of

Table 1

\begin{tabular}{|c|c|c|c|c|c|c|c|c|c|c|c|}
\hline Period (days) (110 days) & $\mathrm{B}(10)$ & $\mathrm{P}(10)$ & $3 \mathrm{mg}(10)$ & W (10) & $6 \mathrm{mg}(10)$ & W (10) & $9 \mathrm{mg}(10)$ & W (10) & $12 \mathrm{mg}(10)$ & W (10) & $15 \mathrm{mg}(10)$ \\
\hline Melatonin & - & - & $\mathrm{x}$ & - & $\mathrm{x}$ & - & $\mathrm{x}$ & - & $\mathrm{x}$ & - & $\mathrm{x}$ \\
\hline Actigraphy & $\mathrm{x}$ & $\mathrm{x}$ & $\mathrm{x}$ & $\mathrm{x}$ & $\mathrm{x}$ & $\mathrm{x}$ & $\mathrm{x}$ & $\mathrm{x}$ & $\mathrm{x}$ & $\mathrm{x}$ & $\mathrm{x}$ \\
\hline Pittsburgh test (end of each period) & $\mathrm{x}$ & $\mathrm{x}$ & $\mathrm{x}$ & $\mathrm{x}$ & $\mathrm{x}$ & $\mathrm{x}$ & $\mathrm{x}$ & $\mathrm{x}$ & $\mathrm{x}$ & $\mathrm{x}$ & $\mathrm{x}$ \\
\hline Urine collection (end of each period) & $\mathrm{x}$ & $\mathrm{x}$ & $\mathrm{x}$ & $\mathrm{x}$ & $\mathrm{x}$ & $\mathrm{x}$ & $\mathrm{x}$ & $\mathrm{x}$ & $\mathrm{x}$ & $\mathrm{x}$ & $\mathrm{x}$ \\
\hline Blood collection & $\mathrm{x}$ & - & - & - & - & - & - & - & - & - & $\mathrm{x}$ \\
\hline
\end{tabular}

B, basal period; , placebo period; $\mathrm{W}$, washout period. 
each different dose of melatonin and washout periods. The samples were stored at $-80{ }^{\circ} \mathrm{C}$ until biochemical assay. The total antioxidant capacity in the urinary samples was quantified using a commercial Antioxidant assay kit (Item No.709001, Cayman, Michigan, USA), with a plate-based colorimetric measurement of $405 \mathrm{~nm}$, according to the manufacturer's instructions.

\section{Serum collection and TAC in serum samples}

Blood samples were drawn from all volunteers before the beginning of the trial (basal values) and after the administration of last dose of $15 \mathrm{mg}$ of melatonin, at $8.00 \mathrm{~h}$. The samples were transferred unheparinized to a pre-prepared tube containing serum-separating gel, and were then centrifuged at room temperature for $30 \mathrm{~min}$ at $600 \mathrm{~g}$. The serum was then divided into aliquots in Eppendorf vials, and kept frozen at $-80^{\circ} \mathrm{C}$ until the time of assay.

The TAC in the serum samples were quantified following the method described by Turoli et al. (2004), with slight modifications.

\section{Statistical analysis}

Kolmogorov-Smirnov test was applied for examining normality of the distribution of the results. As normality could be assumed,
Analysis of Variance (ANOVA), followed by Tukey's post-hoc test to compare between groups was used. Unpaired T-test was used for analyze the TAC in serum samples. Values are presented as mean \pm SEM of the number of determinations. The degree of significance was set at $p<0.05$. All analyses were performed using the statistical package GraphPad Prism (version 5.0, 2007; GraphPad Software, Inc; San Diego, CA).

The study was not planned with a prospective power calculation. Nevertheless, power was computed retrospectively based on the observed value of the effect size. Thus, a post hoc power analysis was carried out using $G^{*}$ Power (version 3.1.5) (Faul et al., 2007), and the effect size (Cohen's d) was reported, with a dvalue $>0.5$ indicating a large effect size.

\section{Results}

Sleep values of seven different sleep parameters (sleep latency, total nocturnal activity, wake bouts, sleep efficiency, immobility, actual sleep time and assumed sleep) obtained during the intake of either melatonin or a placebo period in all participants are shown in Fig. 1.

In general, the administration of melatonin caused decreases of sleep latency (Fig. 1A) and total nocturnal activity (Fig. 1B). In particular, the intake of $9 \mathrm{mg}, 12 \mathrm{mg}$ and $15 \mathrm{mg} /$ day were effective
A

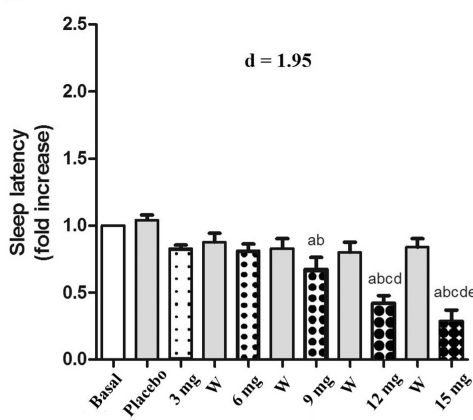

D 2

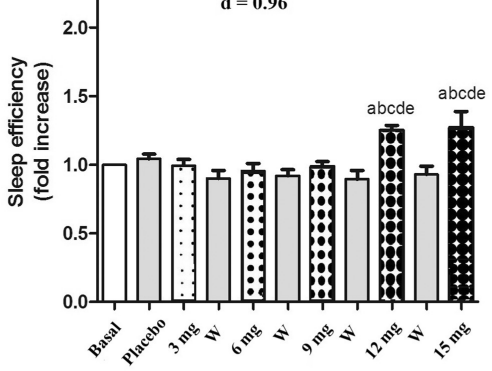

$\mathbf{F}$

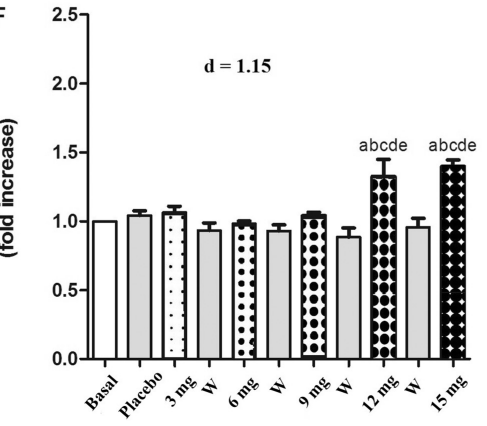

B

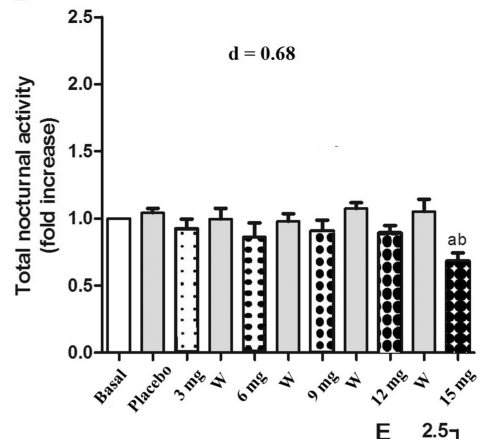

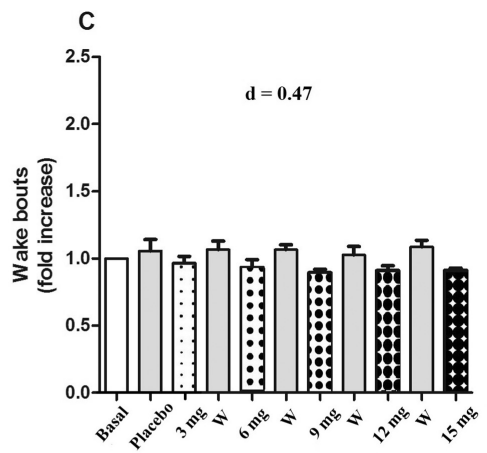

$\begin{array}{ll}E & 2.5 \\ \mathrm{~d}=1.59\end{array}$

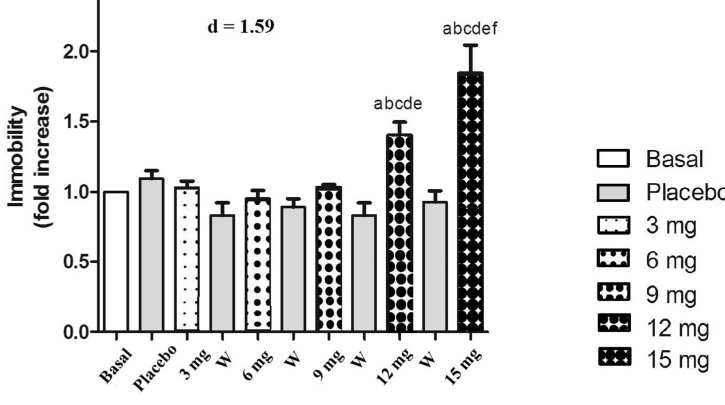

G

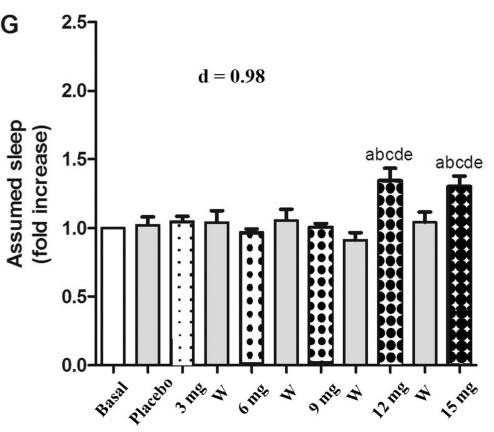

Fig. 1. Effect of the administration of different doses of melatonin (3, 6, 9, 12, $15 \mathrm{mg} /$ day during 10 days each dose) in different parameters obtained by actigraphy: sleep latency (1A), total nocturnal activity (1B), wake bouts (1C), sleep efficiency (1D), immobility (1E), actual sleep time (1F), assumed sleep (1G). Bars indicate mean scores \pm SEM from 33 participants. ${ }^{a} P<0.05$ with respect to the basal value; ${ }^{b} P<0.05$ with respect to the placebo; ${ }^{\mathrm{C}} P<0.05$ with respect to $3 \mathrm{mg} ;{ }^{\mathrm{d}} P<0.05$ with respect to $6 \mathrm{mg}$; ${ }^{\mathrm{e}} P<0.05$ with respect to $9 \mathrm{mg}$ and ${ }^{\mathrm{f}} \mathrm{P}<0.05$ with respect to $12 \mathrm{mg}$. Effect size (Cohen's d) is reported in each panel. 
to diminish significantly $(p<0.05)$ the sleep latency, whereas $15 \mathrm{mg}$ /day produced not only a significant $(p<0.05)$ decrease in sleep latency, but also in total nocturnal activity, with respect to basal and placebo conditions. Regarding the parameter wake bouts, slight decreases were observed, however no significant changes were reported after the administration of any dose of melatonin.

On the other hand, the intake of 12 and $15 \mathrm{mg}$ /day of melatonin increased $(p<0.05)$ the sleep efficiency (Fig. 1D), the immobility (Fig. 1E), the actual sleep time (Fig. 1F) and the assumed sleep (Fig. 1G) with respect to any of previous doses as well as placebo period.

Moreover, our results show that the administration of melatonin improved the self-rated sleep quality (Fig. 2), decreasing its score. This decrease in the total score of Pittsburgh test was significant $(p<0.05)$ after the intake of $6 \mathrm{mg}, 9 \mathrm{mg}, 12 \mathrm{mg}$ and $15 \mathrm{mg} /$ day of melatonin in relation with either baseline or placebo periods. In addition, significant changes were observed after the administration of $12 \mathrm{mg}$ of melatonin with respect to $3 \mathrm{mg} /$ day of melatonin.

Fig. 3 shows a significant dose-dependent increase $(p<0.05)$ in aMT6-s levels that was found after the consumption of any dose of melatonin $(3,6,9,12$, and $15 \mathrm{mg})$, with respect to basal and placebo values.

Furthermore, it is interesting to note that the administration of $12 \mathrm{mg}$ caused significant $(p<0.05)$ differences with respect to the doses of 3, 6 and $9 \mathrm{mg}$; whereas the intake of $15 \mathrm{mg}$ of melatonin produced significant $(p<0.05)$ changes with respect to $3,6,9$, and $12 \mathrm{mg}$ of melatonin.

Regarding antioxidant capacity, represented in Fig. 4, a dosedependent increase in antioxidant capacity levels in urine was found after the consumption of any dose of melatonin $(3,6,9,12$, and $15 \mathrm{mg})$, with significant $(p<0.05)$ increases after the intake of 9,12 , and $15 \mathrm{mg}$ of melatonin, with respect to basal and placebo values. Interesting to note that the administration of $12 \mathrm{mg}$ of melatonin also produced a significant $(p<0.05)$ increase in this parameter with respect to the effect caused after the ingestion of $3 \mathrm{mg}$ of melatonin; whereas the intake of $15 \mathrm{mg}$ of melatonin produced a significantly $(p<0.05)$ increased in total antioxidant capacity with respect to the previous doses of melatonin assessed.

In the same line, the serum total antioxidant (Fig. 5) was significantly $(p<0.05)$ increased after the intake of the last dose of $15 \mathrm{mg}$ of melatonin, with respect to basal condition.

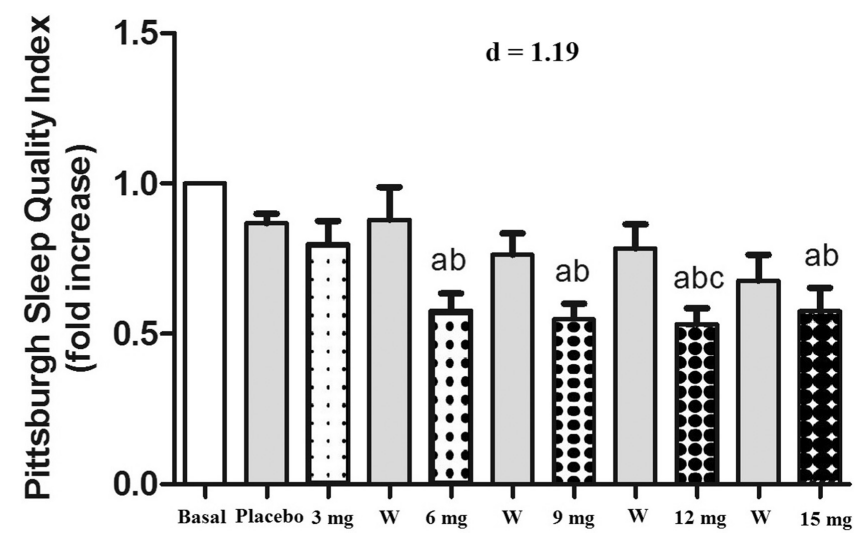

Fig. 2. Effect of the administration of different doses of melatonin (3, 6, 9, 12,15 mg/ day during 10 days each dose) on Pittsburgh Sleep Quality Index (PSQI). Bars indicate mean scores \pm SEM from 33 participants. ${ }^{a} P<0.05$ with respect to the basal value; ${ }^{\mathrm{b}} \mathrm{P}<0.05$ with respect to the placebo; ${ }^{\mathrm{C}} \mathrm{P}<0.05$ with respect to $3 \mathrm{mg}$. Effect size (Cohen's d) is reported in the figure.

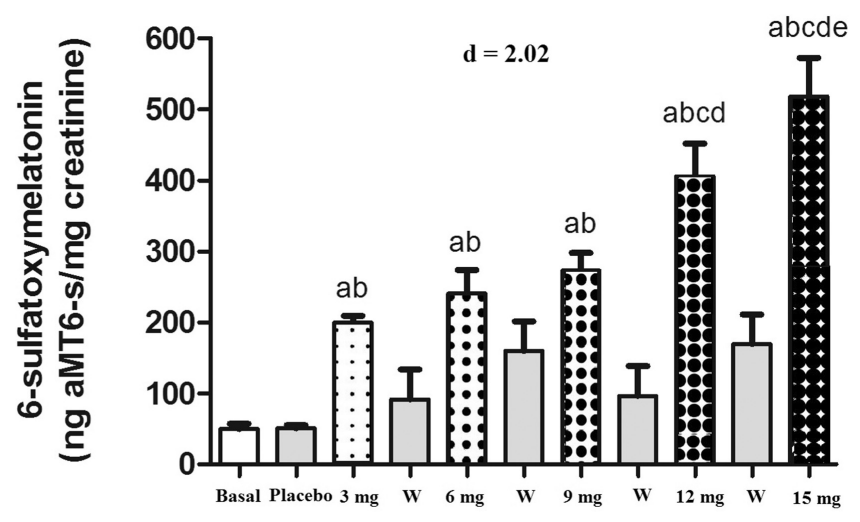

Fig. 3. Effect of the administration of different doses of melatonin (3, 6, 9,12,15 mg/ day during 10 days each dose) on 6-sulfatoxymelatonin (aMT6-s) levels. Bars indicate mean scores \pm SEM from 33 participants. ${ }^{\mathrm{a}} \mathrm{P}<0.05$ with respect to the basal value; ${ }^{\mathrm{b}} \mathrm{P}<0.05$ with respect to the placebo; ${ }^{\mathrm{c}} \mathrm{P}<0.05$ with respect to $3 \mathrm{mg}$; ${ }^{\mathrm{d}} \mathrm{P}<0.05$ with respect to $6 \mathrm{mg}$; ${ }^{\mathrm{e}} \mathrm{P}<0.05$ with respect to $9 \mathrm{mg}$. Effect size (Cohen's d) is reported in the figure.

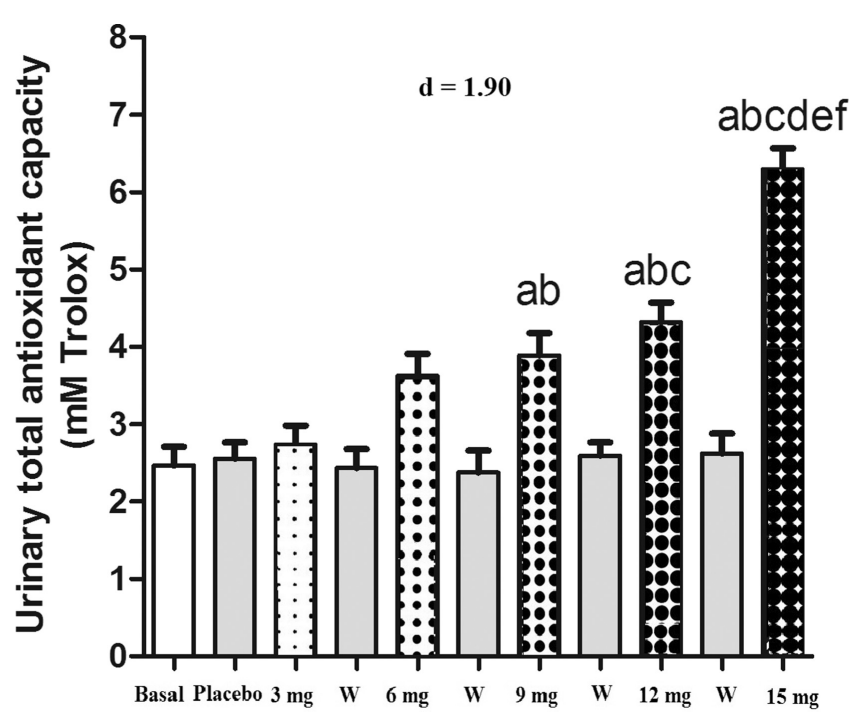

Fig. 4. Effect of the administration of different doses of melatonin $(3,6,9,12,15 \mathrm{mg}$ day during 10 days each dose) on total antioxidant capacity in urine. Bars indicate mean scores \pm SEM from 33 participants. ${ }^{a} P<0.05$ with respect to the basal value; ${ }^{\mathrm{b}} \mathrm{P}<0.05$ with respect to the placebo; ${ }^{\mathrm{C}} \mathrm{P}<0.05$ with respect to $3 \mathrm{mg}$; ${ }^{\mathrm{d}} P<0.05$ with respect to $6 \mathrm{mg}$; ${ }^{\mathrm{P}}<0.05$ with respect to $9 \mathrm{mg}$ and ${ }^{\mathrm{f}} P<0.05$ with respect to $12 \mathrm{mg}$. Effect size (Cohen's d) is reported in the figure.

\section{Discussion}

More than $90 \%$ of patients with FM refer sleep disturbances, including non-restorative sleep, insomnia, early awakenings and poor sleep quality (Chinn et al., 2016). The criteria proposed in 2010 by ACR classification of fibromyalgia (Wolfe et al., 2010) includes the importance of sleep disorders in this group of patients, since an impaired nocturnal sleep is presumably involved in the pathogenesis of the development of pain.

It has been suggested that patients with FM have a lower melatonin secretion during darkness than healthy subjects. It may contribute to impaired sleep at night, non-restorative sleep, fatigue during the day, and changes in pain perception (Wikner et al., 1998), which could be a predisposing factor in the formation of trigger points (Bennet, 2002). In this line, melatonin and its agonist receptor have been used in the management of disorders related to circadian rhythms and pain, such as fibromyalgia. Thus, De Zanette et al. (2014) have proposed that melatonin administration exerts a 


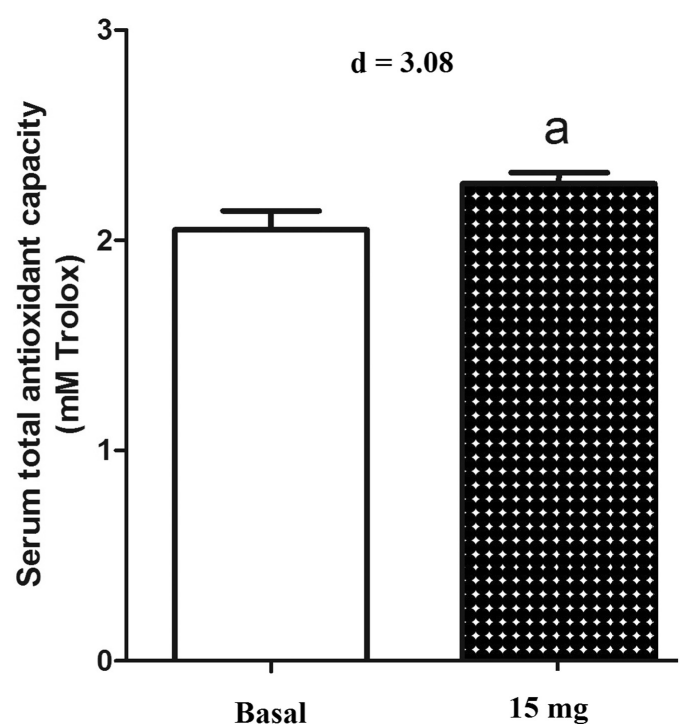

Fig. 5. Effect of the administration of $15 \mathrm{mg}$ of melatonin/day on total antioxidant capacity in serum. Bars indicate mean scores \pm SEM from 33 participants. ${ }^{\text {a }} P<0.05$ with respect to the basal value. Effect size (Cohen's d) is reported in the figure.

direct effect on pain pathways and/or on the levels of signalling chemicals that regulate pain, thereby improving the FM symptoms, such as sleep quality or pain.

Several studies in patients with this syndrome have evaluated the objective sleep quality by actigraphy. The assessment with actigraphy has the advantage of providing objective information on sleep habits (Martin and Hakim, 2011; Sadeh, 2011). In fact, the American Association of Sleep Disorders establishes the use of actigraphy, whose accuracy is $86 \%$, as a valid method of evaluation in specific domains of research of sleep and sleep medicine (Littner et al., 2003). In this line, Korszun et al. (2002) have used this technique to evaluate the nocturnal activity in patients with FM. They observed higher nocturnal activity in these patients compared to healthy subjects. In the present study, it was found a reduction in the nocturnal activity of patients after the intake of $15 \mathrm{mg}$ /day of melatonin during 10 days. However, other authors have suggested that doses of $3 \mathrm{mg} /$ day of melatonin during 30 days (Citera et al., 2000) as well as $5 \mathrm{mg} /$ day during 60 days (Hussain et al., 2011) are enough for improving sleep disturbances. These differences may be related to the clinical profile of the patients, the length of the treatment (30-60 days vs. 10 days) or differences in the experimental design (Rehm et al., 2010). Nevertheless, it is worthy to note that not only $15 \mathrm{mg}$, but also $12 \mathrm{mg}$ of melatonin were effective in improving 5 out of 7 sleep parameters evaluated: sleep latency, sleep efficiency, immobility, actual sleep time and assumed sleep.

The evaluation of sleep parameters by actigraphy offers relevant information for the management of circadian rhythms. Nevertheless, other tools such as the PSQI have been proven to be effective to evaluate the sleep quality in terms of subjectivity both in healthy and sickness population (Buysse et al., 1989; Osorio et al., 2006). Hence, Hita-Contreras et al. (2014) have reported the validation of PSQI for measuring the sleep quality in patients with FM. Since a number of these patients perceive their nocturnal sleep as nonrestorative sleep (Bennet et al., 2007; Moldofsky, 2002), and feel unrefreshed upon awakening, the combination of both techniques offer a better understanding about how the sleep quality is in this syndrome. In the present study, it is interesting to note that despite subjective sleep improved significantly after the intake of $6,9,12$, and $15 \mathrm{mg}$ of melatonin, the sleep parameters measured by actigraphy were, in general, enhanced after the administration of
12 and $15 \mathrm{mg}$ of melatonin. These differences may be due to the fact that melatonin not only exerts its function as a chronobiotic molecule but also it is involved in pain pathways. If melatonin ameliorates pain in patients with FM as previous authors have suggested (Citera et al., 2000; De Zanette et al., 2014), it might be possible that the present population has perceived better quality of sleep with lower doses of melatonin. However, further studies are needed in this direction to clarify this aspect.

On the other hand, the quantification of aMT6-s, a metabolite that is considered to reflect the nocturnal melatonin concentration, may also contribute to improve the subjective sleep perception of these patients. In fact, this metabolite increased after all the doses assayed $(3,6,9,12$, and $15 \mathrm{mg})$. Moreover, it is important to note that better improvements in the sleep parameters evaluated were detected after 12 and $15 \mathrm{mg} /$ melatonin, coinciding with the highest aMT6-s concentration.

It has been proposed that the reduced oxidative capacity participate in the FM pathophysiology (Fatima et al., 2015; Suantawee et al., 2013; Valko et al., 2006). Herein, oxidant/ antioxidant balances may play an essential role in this syndrome. In the present study, the antioxidant capacity status was increased after the ingestion of melatonin. Based on the well-known antioxidant properties of this indolamine, we cannot discard that these effects may be attributed to the elevation of circulatory melatonin levels in these patients. In fact, the highest antioxidant levels were found after the ingestion of $15 \mathrm{mg}$ of melatonin, coinciding with the highest augmentation of aMT6-s levels.

\section{Conclusion}

Taking everything into account, the administration of melatonin improved the sleep quality, increased the aMT6-s levels in a dose-dependent manner, and caused a noticeably enhancement of both urinary and serum total antioxidant levels in patients with FM. Therefore, the use of melatonin as adjuvant therapy might be useful for the management of FM.

\section{Conflict of interests}

The authors declare that they have no conflict of interests.

\section{Compliance with ethical standards}

All procedures performed in studies involving human participants were in accordance with the ethical standards of the institutional and/or national research committee and with the 1964 Helsinki declaration and its later amendments or comparable ethical standards. Informed consent was obtained from all individual participants included in the study.

\section{Acknowledgements}

We are indebted to the volunteers in this study for their outstanding commitment and cooperation.

This work has been financed by Junta de Extremadura European Regional Development Funds (ERDF) grant BBB021GR15051. M. Garrido holds a research post-doctoral fellowship from Gobierno de Extremadura (jointly financed by the European Regional Development Fund (ERDF); ref. PO14013).

\section{References}

Akbas, A., Inanir, A., Benli, I., Onder, Y., Aydogan, L., 2014. Evaluation of some antioxidant enzyme activities (SOD and GPX) and their polymorphisms (MnSOD2 Ala9Val, GPX1 Pro198Leu) in fibromyalgia. Eur. Rev. Med. Pharmacol. Sci. 18, 1199-1203. 
Akkuş, S., Naziroğlu, M., Eriş, S., Yalman, K., Yilmaz, N., Yener, M., 2009. Levels of lipid peroxidation, nitric oxide, and antioxidant vitamins in plasma of patients with fibromyalgia. Cell Biochem. Funct. 27 (4), 181-185.

Bellato, E., Marini, E., Castoldi, F., Barbasetti, N., Mattei, L., Bonasia, D.E., Blonna, D., 2012. Fibromyalgia syndrome: etiology, pathogenesis, diagnosis, and treatment. Pain Res Treat. 2012, 426130.

Bennet, R.M., Jones, J., Turk, D.C., Russel, I.J., Matallana, L., 2007. An internet survey of 2, 596 people with fibromyalgia. BMC Musculoskelet Disord. 8, 27.

Bennet, R.M., 2002. The rational management of fibromyalgia patient. Rheum. Dis. Clin. North Am. 28, 181-199.

Buysse, D.J., Reynolds, C.F., Monk, T.H., Berman, S.R., Kupfer, D.J., 1989. The Pittsburgh Sleep Quality Index: a new instrument for psychiatric practice and research. Psychiatry Res. 28 (2), 193-213.

Chinn, S., Caldwell, W., Gritsenko, K., 2016. Fibromyalgia Pathogenesis and Treatment Options Update. Curr. Pain Headache Rep. 20 (4), 25.

Citera, G., Arias, M.A., Maldonado-Cocco, J.A., Lázaro, M.A., Rosemffet, M.G., Brusco, L.I., et al., 2000. The effect of melatonin in patients with fibromyalgia: a pilot study. Clin. Rheumatol. 19 (1), 9-13.

Cordero, M.D., Alcocer-Gómez, E., Cano-García, F.J., De Miguel, M., Carrión, A.M. Navas, P., Sánchez, J.A., 2011. Clinical symptoms in fibromyalgia are better associated to lipid peroxidation levels in blood mononuclear cells rather than in plasma. PLoS One 6 (10), e26915.

De Zanette, S.A., Vercelino, R. Laste, G., Rozisky, J.R., Schwertner, A., Machado, C. B., et al., 2014. Melatonin analgesia is associated with improvement of the descending endogenous pain-modulating system in fibromyalgia: a phase II, randomized, double-dummy, controlled trial. BMC Pharmacol. Toxicol. 15, 40.

Diaz-Piedra, C., Di Stasi, L.L., Baldwin, C.M., Buela-Casal, G., Catena, A., 2015. Sleep disturbances of adult women suffering from fibromyalgia: a systematic review of observational studies. Sleep Med. Rev. 21, 86-99.

Fatima, G., Das, S.K., Mahdi, A.A., 2015. Some oxidative and antioxidative parameters and their relationship with clinical symptoms in women with fibromyalgia syndrome. Int J. Rheum. Dis. 20, 39-45.

Faul, F., Erdfelder, E., Lang, A.G., Buchner, A., 2007. G*Power 3: a flexible statistical power analysis program for the social, behavioural, and biomedical sciences. Behav. Res. Methods 39, 175-191.

Giacomelli, C., Sernissi, F., Sarzi-Puttini, P., Di Franco, M., Atzeni, F., Bazzichi, L., 2013. Fibromyalgia: a critical digest of the recent literature. Clin. Exp. Rheumatol. 31, S153-S157.

Hita-Contreras, F., Martinez-Lopez, E., Latore-Román, P.A., Garrido, F., Santos, M.A., Martinez-Amat, A., 2014. Reliability and validity of the Spanish version of the Pittsburgh Sleep Quality Index (PSQI) in patients with fibromyalgia. Rheumatol. Int. 34 (7), 929-936.

Hussain, S.A.R., Al-Khalifa, I.I., Jasim, N.A., Gorial, F.I., 2011. Adjuvant use of melatonin for treatment of fibromyalgia. J. Pineal Res. 50, 267-271.

Korszun, A., Young, E.A., Engleberg, N.C., Brucksch, C.B., Greden, J.F., Crofford, L.A. 2002. Use of actigraphy for monitoring sleep and activity levels in patients with fibromyalgia and depression. J. Psychosom. Res. 52 (6), 439-443.
Littner, M., Kushida, C.A., Anderson, W.M., Bailey, D., Berry, R.B., Davila, D.G., et al., 2003. Practice parameters for the role of actigraphy in the study of sleep and circadian rhythms: an update for 2002. Sleep 26 (3), 337-341.

Mahdi, A.A., Fatima, G., Das, S.K., Verma, N.S., 2011. Abnormality of circadian rhythm of serum melatonin and other biochemical parameters in fibromyalgia syndrome. Indian J. Biochem. Biophys. 48 (2), 82-87.

Martin, J.L., Hakim, A.D., 2011. Wrist actigraphy. Chest 139 (6), 1514-1527.

Moldofsky, H., 2002. Management of sleep disorders in fibromyalgia. Rheum. Dis. Clin. N. Am. 28, 353-365.

Nazıroğlu, M., Akkuş, S., Soyupek, F., Yalman, K., Çelik, Ö., Eriş, S., Uslusoy, G.A., 2010 Vitamins $C$ and $E$ treatment combined with exercise modulates oxidative stress markers in blood of patients with fibromyalgia: a controlled clinical pilot study. Stress 13 (6), 498-505.

Osorio, C.D., Gallinaro, A.L., Lorenzi-Filho, G., Lage, L.V., 2006. Sleep quality in patients with fibromialgia using the Pittsburgh Sleep Quality Index. J. Rheumatol. 33 (9), 1863-1865.

Rehm, S.E., Koroschetz, J., Gockel, U., Brosz, M., Freynhagen, R., Tölle, T.R., Baron, R. 2010. A cross-sectional survey of 3035 patients with fibromyalgia: subgroups of patients with typical comorbidities and sensory symptom profiles. Rheumatology 49 (6), 1146-1152.

Sánchez, A., Calpena, A.C., Clares, B., 2015. Evaluating the oxidative stress in inflammation: role of melatonin. Int. J. Mol. Sci. 16 (8), 16981-17004

Sadeh, A., 2011. The role and validity of actigraphy in sleep medicine: an update. Sleep Med. Rev. 15 (4), 259-267.

Sartori, J.E.T., Cireia, L.F., Martins, D.P., Rocha, G.P., Cintra, J.R., 2016. Association of markers of oxidative stress, medication therapy and life habits in fibromyalgia. J. Rheum. Dis. Treat. 2, 35.

Spaeth, M., Rizzi, M., Sarzi-Puttini, P., 2011. Fibromyalgia and sleep. Best Pract. Res. Clin. Rheumatol. 25 (2), 227-239.

Suantawee, T., Tantavisut, S., Adisakwattana, S., Tanavalee, A., Yuktanandana, P., Anomasiri, W., et al., 2013. Oxidative stress, vitamin e, and antioxidant capacity in knee osteoarthritis. J. Clin. Diagn. Res. 7 (9), 1855-1859.

Tan, D.X., Chen, L.D., Poeggeler, B., Manchester, L.C., Reiter, R.J., 1993. Melatonin: potent, endogenous hydroxyl radical scavenger. Endocr. J. 1 (4), 57-60.

Turoli, D., Testolin, G., Zanini, R., Bellù, R., 2004. Determination of oxidative status in breast and formula milk. Acta Paediatr. 93, 1569-1574.

Valko, M., Rhodes, C.J., Moncol, J., Izakovic, M.M., Mazur, M., 2006. Free radicals, metals and antioxidants in oxidative stress-induced cancer. Chem. Biol. Interact. 160 (1), 1-40.

Wikner, J., Hirsch, U., Wetterberg, L., Röjdmark, S., 1998. Fibromyalgia: a syndrome associated with decreased nocturnal melatonin secretion. Clin. Endocrinol. 49 (2), 179-183.

Wilhelmsen, M., Amirian, I., Reiter, R.J., Rosenberg, J., Gögenur, I., 2011. Analgesic effects of melatonin: a review of current evidence from experimental and clinical studies. J. Pineal Res. 51 (3), 270-277.

Wolfe, F., Clauw, D.J., Fitzcharles, M.A., Goldenberg, D.L., Katz, R.S., Mease, P., et al., 2010. The American College of rheumatology preliminary diagnostic criteria for fibromyalgia and measurement of symptom severity. Arthritis Care Res. 62 (5), 600-610. 